\title{
Comparison of the efficacy of ShotBlocker and cold spray in reducing intramuscular injection-related pain in adults
}

\author{
A prospective, randomized, controlled trial
}

Sedat Bilge, MD, Attila Aydin, MD, Cem Gun, MD, Hasan Aldinc, MD, Yahya A. Acar, MD, Serpil Yaylaci, MD,

Orhan Cinar, MD, Veysel Balci, $M D$.

\begin{abstract}

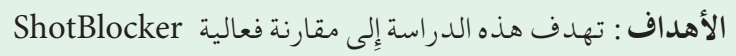

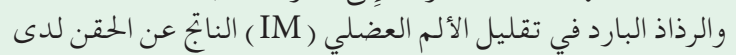

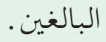

المنهجية: هذه الدراسة مستقبلية عشوائية خاضعة للمراقبة أجريت في الفترة الواقعة بين يناير 2018 ومارس الذبة

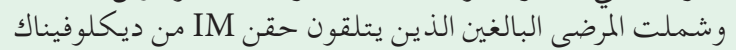

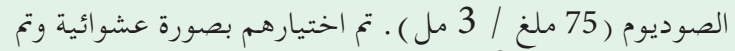

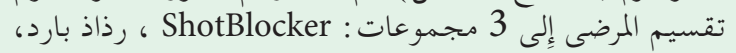

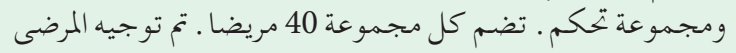

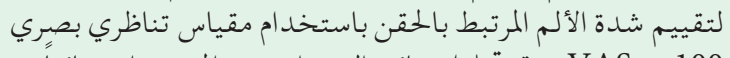

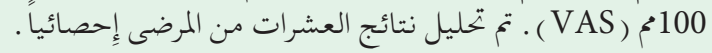

النتائج : كانت درجات VAS أقل في مجموعات ShotBlocker

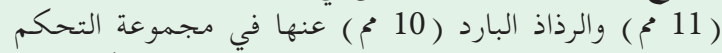

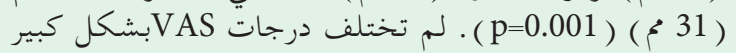

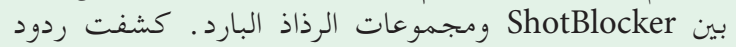
المشغلين أن ShotBlocker كان من الصعب إِدارته أكثر من الرش كُد

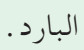

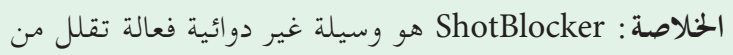
الألم المرتبط بالحقن العضلي IM وتشبه فعاليته فعالية الرذاذ

Objectives: To compare the efficacy of ShotBlocker and cold spray in reducing intramuscular (IM) injection-related pain in adults.

Methos: A prospective, randomized, controlled study carried out between January 2018 and March 2018 at the Department of Emergency Medicine, Acibadem Mehmet Ali Aydinlar University, School of Medicine, Istanbul, Turkey. Adult patients receiving IM injection of diclofenac sodium $(75 \mathrm{mg} / 3 \mathrm{ml})$ were included. The patients were randomized into 3 groups: ShotBlocker, cold spray, and control. Each group comprised 40 patients. Patients were instructed to rate the intensity of IM injection-related pain using a $100-\mathrm{mm}$ visual analog scale (VAS). Visual analog scale scores of the patients were statistically analyzed.

Results: Visual analog scale scores were lower in the ShotBlocker $(11 \mathrm{~mm})$ and cold spray $(10 \mathrm{~mm})$ groups than in the control group ( $31 \mathrm{~mm})(p=0.001)$. There were no significant differences in VAS scores between the ShotBlocker and cold spray groups. The operators' responses revealed that ShotBlocker was more difficult to administer than cold spray.

Conclusion: ShotBlocker is an effective nonpharmacological method that reduces IM injectionrelated pain and is similar in efficacy, to cold spray.

Saudi Med J 2019; Vol. 40 (10): 996-1002 doi: 10.15537/smj.2019.10.24322

From the Department of Emergency Medicine (Bilge, Acar), Gulhane School of Medicine, University of Health Sciences, Ankara; from the Department of Aesthetics (Aydin), Miaclinics Health \& Beauty Clinic, Metropol İtanbul Residence Tower, Atasehir, Istanbul; and from the Department of Emergency Medicine (Gun, Aldinc, Yaylaci, Cinar, Balci) Acibadem Mehmet Ali Aydinlar University, School of Medicine, Istanbul, Turkey.

Received 25th June 2019. Accepted 15th September 2019.

Address correspondence and reprint request to: Dr. Yahya A. Acar, Department of Emergency Medicine, Gulhane Medical School, Health Sciences University, Ankara, Turkey. E-mail: yahyaacar@gmail.com ORCIDF ID: https://orcid.org/0000-0002-9572-5738

Disclosure. Authors have no conflict of interests, and the work was not supported or funded by any drug company. 
$\mathrm{I}$ ntramuscular (IM) injection is an invasive hospital intervention that causes substantial pain. ${ }^{1}$ Intramuscular may impair adherence of patients to treatment. ${ }^{2}$ Intramuscular injection-related pain results from mechanical trauma and a sudden increase in pressure, owing to the introduction of the needle and administration of the substance directly into the muscle. Previous studies have investigated the pharmacological and non-pharmacological methods that reduce or prevent this type of pain. ${ }^{3}$ The major pharmacological methods for reducing IM injection-related pain include the administration of topical anesthetic agents and application of cold spray. The use of topical anesthetics is limited in the emergency department (ED) due to low and slow analgesic effects, risk of systemic toxicity, and local side effects. ${ }^{4,5}$ Cold sprays effectively reduce pain, are safe for both healthcare providers and patients, and do not cause local tissue injuries. ShotBlocker is a plastic device with several short and blunt contact points on its surface that contact the patient's skin. Non-pharmacological methods such as cold spray and ShotBlocker (Bionix, Toledo, Ohio) reduce pain via gate control theory during the administration of IM injections. The use of the ShotBlocker device has no reported side effects. ${ }^{6-9}$

Cold sprays are a well-established method used to reduce IM injection pain. We thus aimed to evaluate the efficacy of ShotBlocker and cold spray in reducing IM injection-related pain in adult patients. The primary endpoint of the study was the pain scores obtained after injection in ShotBlocker, cold spray, and noninterventional groups. We aimed to evaluate whether the ShotBlocker was user friendly, and we thus considered the secondary endpoint as the operators' perceptions on the use of ShotBlocker and cold spray.

Methods. This study was conducted on 120 patients between January 2018 and March 2018 in the Department of Emergency Medicine, Acibadem Mehmet Ali Aydinlar University, School of Medicine, Istan bul, Turkey. Ethics committee approval has been received from the institution (decision number 2017-11/2). The study was guided by the Declaration of Helsinki and its latest amendments. The center was a tertiary care hospital with 60,000 emergency admissions annually.

Adult patients who were admitted to the ED due to either the administration of already prescribed medications (diclofenac sodium, $75 \mathrm{mg} / 3 \mathrm{ml}$ ) or those who were to receive an IM injection as ordered by the

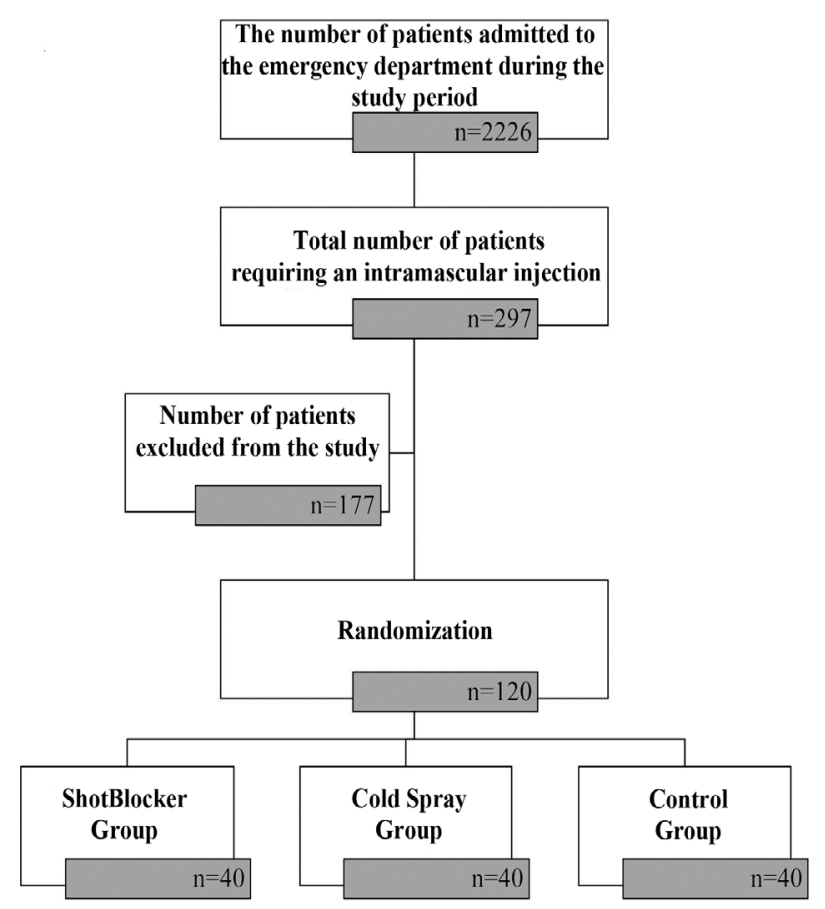

Figure 1 - Flow diagram of the study.

ED physician, participated in the study. They were randomized into 3 study groups, each consisting of 40 patients. The flowchart of the study is presented in Figure 1.

The inclusion criteria were patients aged 18 years or more who signed the informed consent form and were admitted to the ED for administration of prescribed medications or who were scheduled to receive an IM injection as ordered by the ED physician.

The exclusion criteria of the study were pregnant women, those with mental retardation and hearing or vision problems, those presenting to the ED with complaints of anxiety or trauma (soft tissue traumas, multiple traumas, or bone fractures due to traffic accidents or falls), those who experienced alterations in consciousness, those with clinical conditions requiring emergency interventions, secondary injuries, and infections at the site of injection, those who received a previous injection in the same area within the last 24 hours (h), those who experienced failure in the injection procedure at the first attempt, and those who declined to provide informed consent for the study.

The database on ShotBlocker was searched on PubMed and Google Scholar Academic. There were 7 studies that have been conducted on Shotblocker. We 
reviewed these studies to obtain efficacy and safety data on ShotBlocker.

Prior to the study, the investigators conducted a 2-h theoretical and practical training on 2 separate groups of emergency service nurses $(n=14)$ regarding the study protocol and instructions on the use of ShotBlocker (Bionix, Toledo, Ohio) and cold spray (Anticare Cold Spray, BSN Medical Limited, the UK).

Adult patients who approved the informed consent form were randomized into 3 groups: the ShotBlocker group, cold spray group, and control group. The randomization list was generated via block randomization. ${ }^{10}$

When $90 \%$ power and 0.05 2-sided margin of error were assumed, 27 patients were included in each group based on a difference in 16- $\mathrm{mm}$ visual analog scale (VAS) score and $18-\mathrm{mm}$ standard deviation, as reported in previous studies. The study has been conducted on 120 patients and 3 groups each comprising 40 patients. ${ }^{11}$

Standard IM injection procedure. As the gluteal region is confined to the limits defined by the iliac crest, coccyx, and gluteal folds, it was arbitrarily divided into 4 equal-sized areas, among which the upper outer quadrant was considered the site of IM injection. The patient was placed in a prone position and instructed to rotate their feet inward, with the big toes of either foot opposing each other. The injection area was undressed appropriately, and muscle relaxation was examined via palpation. The area of intervention was sterilized with $2 \%$ chlorhexidine solution as preparation for the procedure. All injections were standardized and were performed by emergency department nurses. The medication was administered within 15 second (sec), at a rate of one $\mathrm{ml} / 5 \mathrm{sec}$. Intramuscular injections were performed using a standardized method in which a green catheter ( 21 gauge, 1.5 inches) and a $5-\mathrm{ml}$ syringe were used to administer the medication at a $90^{\circ}$ angle at the injection site.

Control group. The standard IM injection procedure was applied to this group.

ShotBlocker group. In this group, the device was placed just below the intervention site prior to injection with its opening facing upward. The injection was immediately performed with the dominant hand after the device was pressed firmly against the skin of the patient with the non-dominant hand of the operator (Figure 2).

Cold spray group. In this group, injection was performed after administering the cold spray (Anticare, BSN Medical Ltd., East Yorkshire, UK) to the site of injection with a distance of approximately $15 \mathrm{~cm}$

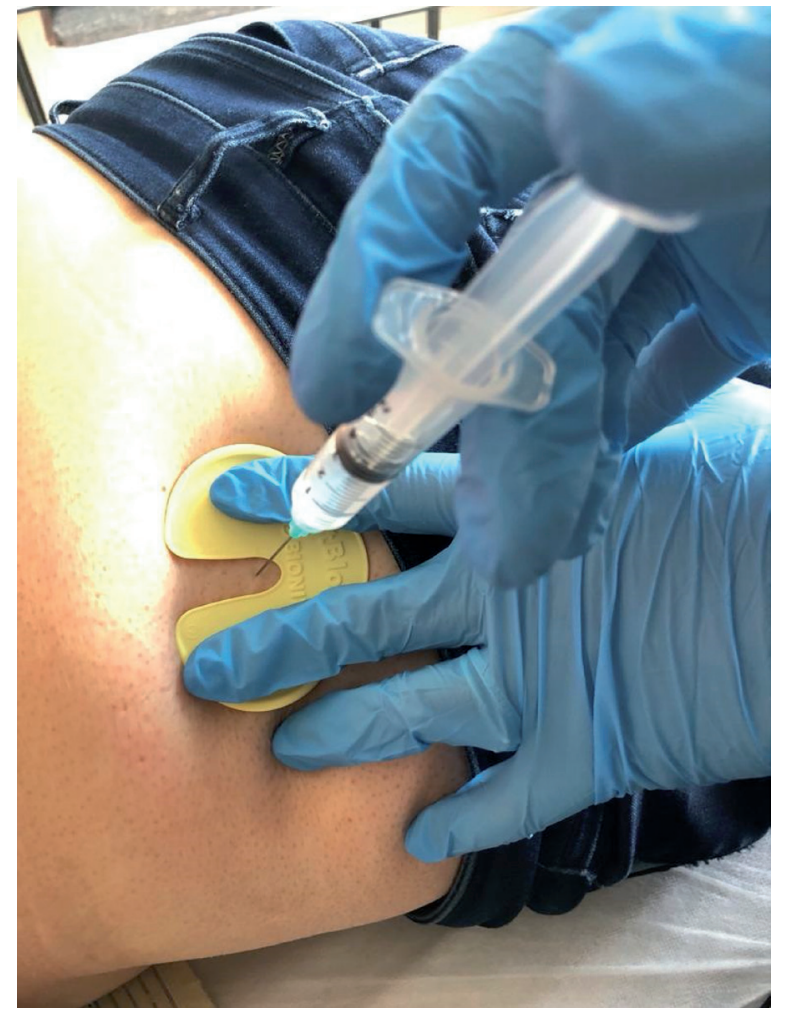

Figure 1 - Intramuscular injection with the use of ShotBlocker.

perpendicularly. No waiting time was required for cold spray use. Operators performed the injection immediately after using cold spray.

The demographic data of the patients, including gender, age, weight, and fear of injection were recorded.

After successfully completing the procedure, the patients were instructed to label the intensity of injection-related pain using a 100-mm-long VAS for pain. A score of 0 in the VAS indicated the absence of pain, whereas a score of 100 indicated the highest level of pain intensity. The patient's skin properties (thin/not thin) were recorded. In addition, whether the patient moved their leg during the procedure (moved/kept stable) was recorded by the operator. The user evaluated the difficulty of administering either the ShotBlocker or cold spray using a 5-point Likert scale.

Statistical analysis. All statistical tests were performed using IBM SPSS Statistics for Windows, version 18.0 (SPSS Inc., Chicago, IL., USA). Descriptive statistics are presented as numbers and percentages for categorical variables. Numerical variables are presented as mean, standard deviation, median, minimum, maximum, 25 th percentile, and 75 th percentile. The conformity of the variables to normal distribution was examined with 
the Kolmogorov-Smirnov test. For categorical variables, Chi-square test was used in multiple comparisons when Chi-square condition was met, whereas Fisher's exact test when Chi-square condition was not met. Spearman correlation analysis was performed to determine the relationship among normally distributed numerical variables. Multiple group comparisons were performed with analysis of variance (ANOVA) if the data were distributed normally. Kruskal-Wallis test was used for analyzing non-normally distributed data. Post hoc analyses were performed with the Mann-Whitney $\mathrm{U}$ test using the Bonferroni correction. The effects of independent risk factors on VAS scores were analyzed using linear regression analysis. The initial candidate risk factors for regression model were selected according to the expert authors' opinion. The VAS scores were evaluated for normal distribution extensively using Shapiro-Wilk test, QQ-detrended plot, histogram graph, skewness-kurtosis, and coefficient of variation being below $30 \%$. The linearity between VAS scores and risk factors were evaluated visually using scatterplots and quantitatively using the Pearson correlation analyses for numerical variables or student t-tests for dichotomous dummy variables, where appropriate. The model fit was analyzed using residual analyses and goodness-of-fit statistics. The residuals were evaluated for normal distribution and homoscedasticity. The results of the linear regression analyses were summarized using parameter coefficients and statistical significance level. The upper limit of type-I error level for statistical significance ( $p$-value) was regarded as 5\% $(p<0.05)$.

Results. Demographic data (including gender, age, and body weight), fear of injection, and skin characteristics were similar in all 3 groups (Table 1). The VAS scores were lower in the ShotBlocker $(11 \mathrm{~mm})$ and cold spray $(10 \mathrm{~mm})$ groups than in the control group $(31 \mathrm{~mm})(p=0.001)$. The VAS scores of the cold spray

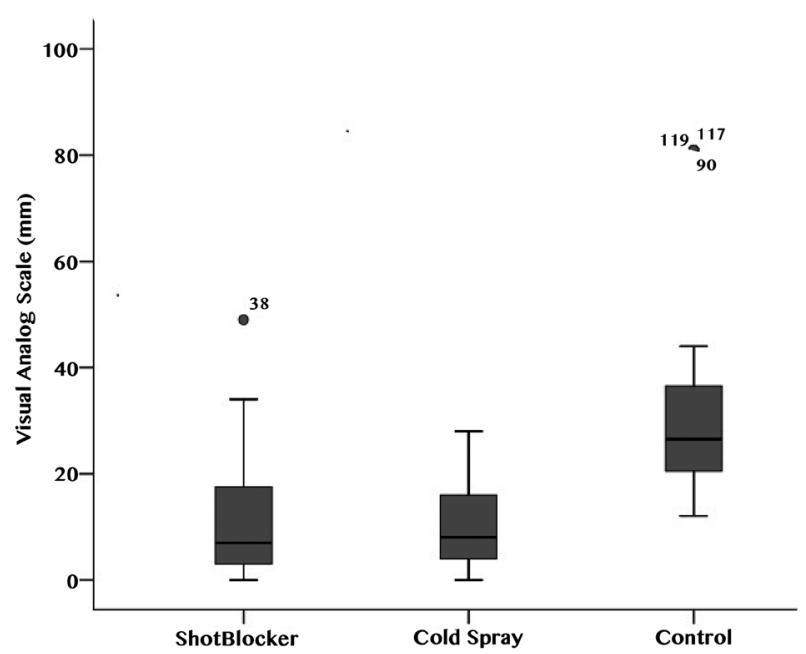

Figure 3 - Mean pain scores of the ShotBlocker, cold spray, and control groups.

Table 1 - Intergroup analysis of the demographic data and clinical characteristics of study groups.

\begin{tabular}{|c|c|c|c|c|}
\hline Variables & ShotBlocker group & Cold spray group & Control group & $P$-value \\
\hline \multicolumn{5}{|l|}{ Gender (n) } \\
\hline Male & 12 & 15 & 22 & \multirow{2}{*}{$0.066^{*}$} \\
\hline Female & 28 & 25 & 18 & \\
\hline \multicolumn{5}{|l|}{ Age } \\
\hline Mean \pm SD & $44 \pm 18$ & $41 \pm 14$ & $38 \pm 13$ & \multirow{2}{*}{$0.292^{\dagger}$} \\
\hline Minimum-maximum & $(32-54)$ & $(31-48)$ & $(29-42)$ & \\
\hline \multicolumn{5}{|l|}{ Weight } \\
\hline Mean \pm SD & $73.53 \pm 15.4$ & $73.43 \pm 11.97$ & $72.75 \pm 11.69$ & \multirow{2}{*}{$0.960^{\ddagger}$} \\
\hline Minimum-maximum & $(60-85.5)$ & $(65-80)$ & $(65-80)$ & \\
\hline \multicolumn{5}{|l|}{ Visual analog scale } \\
\hline Mean $\pm S D$ & $11.48 \pm 11.80$ & $10.20 \pm 8.12$ & $31.58 \pm 16.46$ & \multirow{2}{*}{$<0.001^{\dagger}$} \\
\hline Minimum-maximum & $(3-17.50)$ & $(4-16)$ & $(20.50-3.50)$ & \\
\hline \multicolumn{5}{|l|}{ Fear of injection (n) } \\
\hline None & 23 & 25 & 16 & \multirow{2}{*}{$0.106^{*}$} \\
\hline Present & 17 & 15 & 24 & \\
\hline \multicolumn{5}{|l|}{ Characteristics of skin (n) } \\
\hline Thin & 5 & 6 & 7 & \multirow{2}{*}{$0.822^{*}$} \\
\hline Not thin & 35 & 34 & 33 & \\
\hline \multicolumn{5}{|c|}{ Leg movements during injection (n) } \\
\hline Kept Stable & 38 & 39 & 37 & \multirow{2}{*}{$0.871^{\S}$} \\
\hline Moving & 2 & 1 & 3 & \\
\hline
\end{tabular}


Table 2 - Group-based Statistics for visual analog scale.

\begin{tabular}{|c|c|c|c|c|c|c|c|c|c|}
\hline \multirow{2}{*}{ Parameters } & \multicolumn{3}{|c|}{ ShotBlocker group } & \multicolumn{3}{|c|}{ Cold spray group } & \multicolumn{3}{|c|}{ Control group } \\
\hline & Mean \pm SD & $\mathrm{n}^{\circ}$ & $P$-value* & Mean \pm SD & n & $P$-value* & Mean \pm SD & $\mathrm{n}$ & $P$-value* \\
\hline \multicolumn{10}{|l|}{ Gender } \\
\hline Male & $8.67 \pm 7.41$ & 12 & \multirow{2}{*}{0.534} & $9.13 \pm 7.89$ & 15 & \multirow{2}{*}{0.475} & $30.55 \pm 13.76$ & 22 & \multirow{2}{*}{0.967} \\
\hline Female & $12.68 \pm 13.19$ & 28 & & $10.84 \pm 8.35$ & 25 & & $32.83 \pm 19.61$ & 18 & \\
\hline \multicolumn{10}{|c|}{ Fear of injection } \\
\hline None & $6.48 \pm 6.55$ & 23 & \multirow{2}{*}{0.003} & $5.64 \pm 4.53$ & 25 & \multirow{2}{*}{$<0.001$} & $25.44 \pm 7.17$ & 16 & \multirow{2}{*}{0.079} \\
\hline Present & $18.24 \pm 14.01$ & 17 & & $17.8 \pm 7.04$ & 15 & & $35.67 \pm 19.55$ & 24 & \\
\hline \multicolumn{10}{|c|}{ Characteristics of the skin } \\
\hline Thin & $5 \pm 4.36$ & 5 & \multirow{2}{*}{0.219} & $8 \pm 5.14$ & 6 & \multirow{2}{*}{0.805} & $31.14 \pm 22$ & 7 & \multirow{2}{*}{0.285} \\
\hline Not thin & $12.40 \pm 12.27$ & 35 & & $10.59 \pm 8.54$ & 34 & & $31.67 \pm 15.47$ & 33 & \\
\hline
\end{tabular}

and ShotBlocker group were found similar to each other (Figure 3).

No significant differences were observed in VAS scores according to gender and skin characteristics among the study groups (Mann-Whitney $U$ test; $p=0.467$ and $p=0.408$ ). In the ShotBlocker and cold spray groups, the VAS scores were significantly higher in patients with fear of injection than in those without (Table 2). When the VAS scores of the 3 groups were examined according to age and body weight, no significant differences were observed (Table 3).

Operator difficulties in using ShotBlocker were reported by the users as follows: "it was not difficult at all" ( $n=8 / 40)$, "mildly difficult" ( $n=16 / 40)$, "moderately difficult" ( $n=11 / 40)$, and "very difficult" $(n=5 / 40)$. Operator difficulties associated with the use of cold spray were reported by the users as follows: "not difficult" $(\mathrm{n}=37 / 40)$ and "slightly difficult" $(\mathrm{n}=3 / 40)$. Other degrees of difficulty were not selected by operators in the cold spray group.

The linear regression analysis performed to define the risk factors affecting the VAS scores revealed that fear of injection was a significant risk factor increasing the severity of VAS scores both in the ShotBlocker $(p=0.002)$ and cold spray groups $(p<0.001)$. On the other hand, fear of injection was not found as a significant risk factor increasing the VAS scores in the control group $(p=0.061)$. The results of the linear regression analyses of the study groups are presented in Table 4.

Discussion. Different methods that reduce IM injection-related pain in both adult and pediatric patients have been reported. Prior experiences of patients, $\mathrm{pH}$, osmolality, viscosity, speed of injection, and chemical properties of the drug have been associated with this type of pain. ${ }^{7,11}$ The gate control theory was described by Melzack and Wall. ${ }^{12}$ This theory proposes that a painless physical input, such as warm-cold application,
Table 3 - Correlation between age and weight based on visual analog scale scores according to the study groups.

\begin{tabular}{lcc}
\hline Parameter & $\mathrm{n}$ & Correlation coefficient $^{*}$ \\
\hline ShotBlocker group & & \\
Age & 40 & -0.003 \\
Weight (kg) & 40 & 0.154 \\
Cold spray group & & \\
Age & 40 & 0.037 \\
Weight (kg) & 40 & -0.138 \\
Control group & & \\
Age & 40 & 0.009 \\
Weight (kg) & 40 & -0.066 \\
\hline \multicolumn{2}{c}{ *Spearman's rho correlation analysis, n - frequency } \\
\hline
\end{tabular}

Table 4 - Results of the linear regression analysis in the study groups.

\begin{tabular}{lcc}
\hline Study groups & B & $P$-value \\
\hline ShotBlocker ${ }^{\circledR}$ group & & \\
Constant & -8.847 & 0.458 \\
Leg movements during injection & 12.662 & 0.098 \\
Characteristics of the skin & 1.185 & 0.821 \\
Fear of injection & 11.305 & 0.002 \\
Cold Spray Group & & \\
Constant & -8.000 & 0.260 \\
Leg movements during injection & 5.743 & 0.315 \\
Characteristics of the skin & 4.201 & 0.092 \\
Fear of injection & 12.113 & $<0.001$ \\
Control Group & & \\
Constant & 20.463 & 0.199 \\
Leg movements during injection & 5.192 & 0.601 \\
Characteristics of the skin & -0.299 & 0.965 \\
Fear of injection & 10.127 & 0.061 \\
\hline \multicolumn{2}{c}{ B - unstandardized regression coefficient } \\
\hline
\end{tabular}

compression, vibration, acupuncture, or transcutaneous electrical nerve stimulation, closes the gates to painful input in the central nervous system, thereby preventing painful stimuli. This method can alternatively induce stimuli that inhibit pain sensations. In ShotBlocker and cold spray applications, the gate control theory may 
underscore the mechanisms of action by which IM injection-related pain is attenuated.

ShotBlocker can be used as a non-pharmacological method in the ED during injections for reducing pain. ShotBlocker is a plastic material that is compressed to the site of injection during the procedure, and the injection is performed through the space at the center. The ShotBlocker has several short and blunt contact points on its surface which contact the patient's skin. When the device is pressed against the skin, these contact points stimulate the small-diameter, fast-conducting nerve fibers. However, the requirement for pressing ShotBlocker with one hand has been considered a complicating factor.

In the study by Celik et $\mathrm{al}^{7}{ }^{7}$ the effects of ShotBlocker in reducing pain associated with IM injection of diclofenac sodium, VAS scores were lower in the ShotBlocker arm $(7 \mathrm{~mm})$ than placebo $(20 \mathrm{~mm})$ and control $(26 \mathrm{~mm})$ arms. Our study was conducted on a population with identical characteristics. Our data were compatible with the results of Çelik et al. ${ }^{7}$ Both studies indicated that ShotBlocker was an effective method to reduce pain caused by IM injection of diclofenac sodium. Furthermore, our study demonstrated that the use of either the cold spray or ShotBlocker reduced IM injection-related pain. No side effects were observed in any group. ShotBlocker was found to be more difficult to apply than the cold spray.

Mawhorter et $\mathrm{al}^{13}$ have applied cold sprays or $+4^{\circ} \mathrm{C}$ saline-impregnated cool cotton balls as placebo at the site of injection to compare the effects of these distinct applications on IM injection-related pain during vaccine administrations. The study concluded that the administration of topical cold spray prevented pain. According to the results of our study, the administration of cold spray contributed to reduction of IM injectionrelated pain intensity. No complications were observed during the application of cold spray in our study. However, Mawhorter et $\mathrm{al}^{13}$ reported flammability and fluorocarbon content as limiting factors of cold spray administration. Since both ShotBlocker and cold spray effectively reduce pain, ShotBlocker is the preferable option as it is not constrained by any limiting factors.

Irkoren et $\mathrm{a}^{14}$ reported that a significant reduction in the intensity of pain induced by botulinum toxin injections on the forehead was achieved with the use of a topical anesthetic cream and ethyl chloride-containing cold sprays. Similarly, cold spray application reduces the intensity of pain in arteriovenous fistula procedures as effectively as a topical anesthetic cream. ${ }^{15}$ Moreover, cold sprays decrease the intensity of IM injection-related pain in pediatric patients. ${ }^{16}$ The results of this study are in accordance with the results of studies conducted on different populations comprising different age and race groups. ${ }^{5,9,16}$ These studies have collectively demonstrated that cold sprays can reduce the intensity of injectionrelated pain without causing any complications, and that they are effective in different age groups and body regions when used in different interventional procedures. Nevertheless, patients' allergies should be taken into consideration when adopting this approach.

In a randomized controlled study comparing the ShotBlocker and control groups, Caglar et a ${ }^{17}$ evaluated the perception of pain in neonates during IM injections of hepatitis B vaccine. Pain scores in the ShotBlocker group $(1.64 \pm 0.80)$ during IM injections were lower $(p<0.001)$ than control group $(2.96 \pm 0.73)$, indicating that the use of ShotBlocker reduced the intensity of acute pain. ${ }^{17}$ In our study, the use of ShotBlocker in the adult group reduced IM injection-related pain; thus, ShotBlocker appears to be effective across different age groups.

Chung et $\mathrm{al}^{18}$ reported that compression applied with the fingers at the site of injection over the deltoid muscle before injections was associated with lower pain intensity scores compared to those for conventional IM injections. Compression with the fingers may adopt the same mechanism in gate control theory as that of the ShotBlocker mechanism.

The relationship between pain and anxiety has been described in the literature, which has revealed that a reduction in the severity of anxiety using nonpharmacological methods may lead to a reduction in pain intensity. ${ }^{19,20}$ A notable finding of our study is that the use of ShotBlocker and cold spray in patients with fear of injection led to a significant increase in VAS scores. Patients with fear of injection may benefit more from an anxiolytic drug than from cold spray or ShotBlocker prior to injection. Further, inclusion in a clinical study may be an anxiogenic factor.

Study limitations. This study was conducted at a single center, which limits its generalizability; this is considered a major limitation. The anxiety scores of the participants were not evaluated, which is another limitation of the study. In addition, the non-pharmacological methods used in the study were not compared with pharmacological methods, and a placebo group was not included.

In conclusion, the use of ShotBlocker and cold spray can be effective in reducing pain during injections. Neither of these methods caused complications; however, the use of ShotBlocker was associated with application difficulties. 
Further studies may be beneficial to facilitate the use of ShotBlocker in routine practice.

Acknowledgment. The authors gratefully acknowledge Editage (www.edditage.com) for English language editing.

\section{References}

1. Kant E, Akpinar RB. The effect of music and the pressure applied on pain induced by intramuscular injection. Int J Caring Sci 2017; 10: 1313-1318.

2. Arslan GG, Ozden D. Creating a change in the use of ventrogluteal site for intramuscular injection. Patient Prefer Adherence 2018; 12: 1749-1756.

3. Ogston-Tuck S. Intramuscular injection technique: an evidence-based approach. Nurs Stand 2014; 29: 52-59.

4. Kumar M, Chawla R, Goyal M. Topical anesthesia. J Anaesthesiol Clin Pharmacol 2015; 3: 450-456.

5. Waibel KH, Katial RK. Effect of topical vapocoolant spray on skin test wheal, flare, and pain responses. Ann Allergy Asthma Immunol 2005; 95: 149-153.

6. Cobb JE, Cohen LL. A randomized controlled trial of the ShotBlocker for children's immunization distress. Clin J Pain 2009; 25: 790-796.

7. Celik N, Khorshid L. The use of ShotBlocker for reducing the pain and anxiety associated with intramuscular injection: a randomized, placebo controlled study. Holistic Nurs Pract 2015; 29: 261-271.

8. Drago LA, Singh SB, Douglass-Bright A, Yiadom MY, Baumann BM. Efficacy of ShotBlocker in reducing pediatric pain associated with intramuscular injections. Am J Emerg Med 2009; 27: 536-543.

9. Gupta NK, Upadhyay A, Dwivedi AK, Agarwal A, Jaiswal V, Singh A. Randomized controlled trial of topical EMLA and vapocoolant spray for reducing pain during DPT vaccination. World J Paediatr 2017; 13: 236-241.

10. Urbaniak GC, Plous S. Research randomizer (Version 4.0). [Updated 2019. Cited 2013 June 22]. Available form: http:// www.randomizer.org/
11. Ozdemir L, Piotanarciota E, Akay BN, Akyol A. Effect of methylprednisolone injection speed on the perception of intramuscular injection pain. Pain Manag Nurs 2013; 14: 3-10.

12. Melzack R, Wall PD. Pain mechanisms: a new theory. Science 1965; 150: 971-979.

13. Mawhorter S, Daugherty L, Ford A, Hughes R, Metzger D, Easley K. Topical vapocoolant quickly and effectively reduces vaccine-associated pain: results of a randomized, single-blinded, placebo-controlled study. J Travel Med 2004; 11: 267-272.

14. Irkoren S, Ozkan HS, Karaca H. A clinical comparison of EMLA cream and ethyl chloride spray application for pain relief of forehead botulinum toxin injection. Ann Plast Surg 2015; 75: 272-274.

15. Celik G, Ozbek O, Yilmaz M, Duman I, Ozbek S, Apiliogullari S. Vapocoolant spray vs lidocaine/prilocaine cream for reducing the pain of venipuncture in hemodialysis patients: a randomized, placebo-controlled, crossover study. Int J Med Sci 2011; 8: 623-627.

16. Cohen Reis E, Holubkov R. Vapocoolant spray is equally effective as EMLA cream in reducing immunization pain in school-aged children. Pediatrics 1997; 100: E5.

17. Caglar S, Buyukyilmaz F, Cosansu G, Caglayan S. Effectiveness of ShotBlocker for immunization pain in full-term neonates: a randomized controlled trial. J Perinatal Neonatal Nurs 2017; 31: 166-171.

18. Chung JW, Ng WM, Wong TK. An experimental study on the use of manual pressure to reduce pain in intramuscular injections. J Clin Nurs 2002; 11: 457-461.

19. Goettems ML, Zborowski EJ, Costa FD, Costa VP, Torriani DD. Nonpharmacologic intervention on the prevention of pain and anxiety during pediatric dental care: a systematic review. Acad Pediatr 2017; 17: 110-119.

20. Kapoor S, White J, Thorn BE, Block P. Patients presenting to the emergency department with acute pain: the significant role of pain catastrophizing and state anxiety. Pain Med 2016; 17 : 1069-1078. 